\title{
Assessing Hexagonal Boron Nitride Crystal Quality by Defect Sensitive Etching
}

\author{
T. Hoffman ${ }^{1}$, Y. Zhang ${ }^{1}$, S. Liu ${ }^{1}$, N. Khan ${ }^{2}$, M.E. Twigg ${ }^{3}$, N.D. Bassim ${ }^{4}$ and J.H. Edgar ${ }^{1, a}$ \\ 1. Dept of Chemical Engineering, Kansas State University, Manhattan, Kansas, USA \\ 2. School of Science and Technology, Georgia Gwinnett College, Lawrenceville, Georgia, \\ USA \\ 3. United States Naval Research Laboratory, Washington, DC, USA \\ 4. Dept of Materials Science and Engineering, JHE 357, McMaster University, Hamilton, Ontario, \\ CANADA
}

Hexagonal boron nitride ( $\mathrm{hBN}$ ), the graphite-like polymorph, has been employed for more than 55 years as powders, ceramics, amorphous films, and deposited fine-grain polycrystalline pyrolytic forms $(\mathrm{pBN})$ in applications that take advantage of its high thermal stability, chemical inertness, high thermal conductivity, low x-ray absorption, high electrical resistivity, and lubricating properties [1-4]. Recently, new applications have been envisioned for $\mathrm{hBN}$ that take advantage of its unique structural, optical and electronic properties. These include nanophotonics [5] exploiting its highly anisotropic optical properties, two dimensional atomically-thin transistors that employ hBN's ultra-smooth surfaces, high resistivity, and lattice matching with graphene [6, 7], deep ultraviolet emission, made possible by hBN's large energy band gap $(5.8 \mathrm{eV})$ and high exciton binding energy [8, 9], and solidstate neutron detectors, which rely on the strong interaction of thermal neutrons with the boron-10 isotope $[10,11]$. These applications require $\mathrm{hBN}$ of high structural perfection; defects such as dislocations create charge traps, scattering centers, and recombination sites that degrade its optical and charge transport properties (mobility and minority carrier lifetimes) [9, 12-14].

Here we develop defect sensitive etching (DSE) to quantify the density of non-basal plane dislocations in hBN single crystals. Single crystals were precipitated from a molten nickel-chromium flux saturated with $\mathrm{hBN}$ at $1500{ }^{\circ} \mathrm{C}$ under 1 bar of flowing $\mathrm{N}_{2}$, followed by slow cooling $\left(2-4{ }^{\circ} \mathrm{C} /\right.$ hour $)$. Etching the crystals in a molten eutectic mixture of $\mathrm{NaOH}$ and $\mathrm{KOH}$ between $450{ }^{\circ} \mathrm{C}$ and $525^{\circ} \mathrm{C}$ for 1 minute produced hexagonal pits on (0001) planes. The morphology and topography of these pits were characterized using optical microscopy, SEM and AFM. All etch pits were oriented in the same direction within individual grains. Three types of hexagonal etch pits formed: pits with inclined side walls, flat-bottom pits, and pits combining these features. From an Arrhenius plot of the log of the etch rate versus the inverse temperature, an activation energy of $60 \mathrm{~kJ} / \mathrm{mol}$ was estimated. Screw and mixed-type threading dislocations were also identified in the crystal bulk using diffraction-contrast transmission electron microscopy (TEM). This work demonstrates that DSE is an effective method for estimating the density of non-basal plane dislocations in hBN [15]. 
References:

[1] R.T. Paine and C.K. Narula, Chem. Rev. 90, 73 (1990).

[2] A.W. Moore, J. Cryst. Growth 106, 6 (1990).

[3] R.T. Perkins, Adv. X-Ray Anal. 33, 625 (1990).

[4] R.S. Pease, Acta Crystallogr. 5, 356 (1952).

[5] S.B. Austerman, S.M. Myron, and J.W. Wagner, Carbon 5, 549(1967).

[6] C.R. Dean, et al, Nat. Nanotechnol. 5, 722 (2010).

[7] B. Arnaud, et al, Phys. Rev. Lett. 96, 932 (2006).

[8] Y. Kubota, et al, Science 317, 932 (2007).

[9] Y. Kubota, et al, Chem. Mater. 20, 1661 (2008).

[10] D.S. McGregor, T.C. Unruh, and W.J. McNeil, Nucl. Instrum. Meth. A 591, 530 (2008).

[11] T.C. Doan, et al, Nucl. Instrum. Meth. A 591, 121 (2015).

[12] J. Huang and Y.T. Zhu, Defect Diffus, Forum 186-7, 1 (2000).

[13] S. Turan and K.M. Knowles, Phys. Status Solidi A 150, 227 (1995).

[14] J.L. Weyher and J.J. Kelly, "Defect-selective etching of semiconductors", Springer Handbook of Crystal Growth, 1453-1476 (2010).

[15] The authors acknowledge Dr. Tina Salquero in University of Georgia for the use of Innova AFM for surface studies. This work was supported by US Department of Homeland Security and National Science Foundation under the Academic Research Initiative program (Award No: 2011DN-077- ARI048-03)

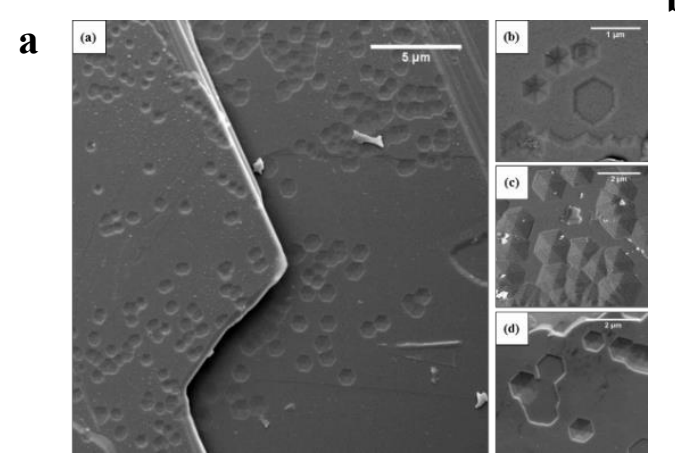

b
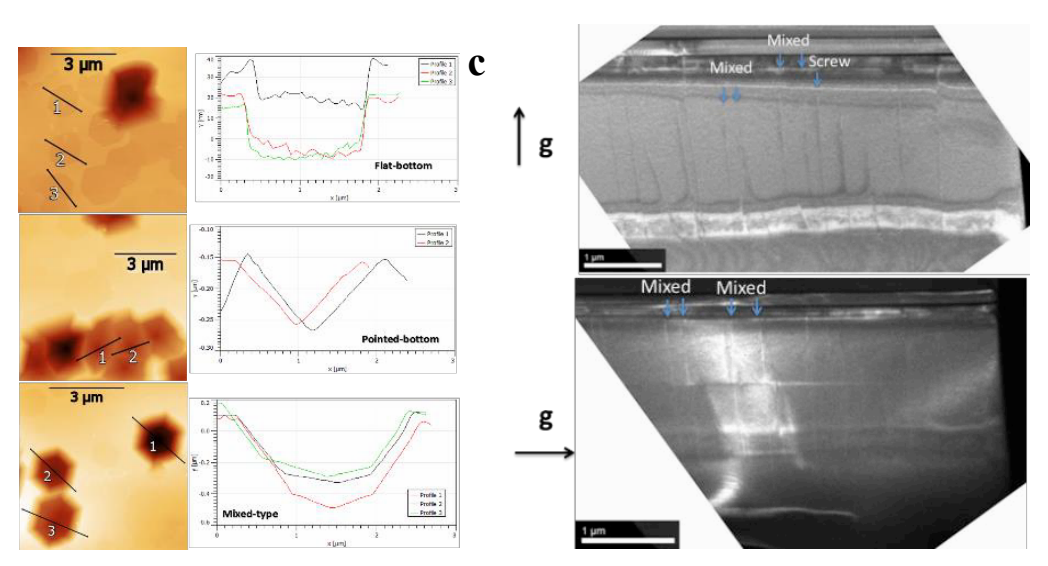

Figure 1. (a) SEM images showing pit shape and distribution on the flakes with different magnifications. (b) AFM images and associated height profiles (from top to bottom) of flat-bottom, pointed bottom and mixed-type etch pits observed on hBN flake. (c) Diffraction-contrast TEM images of hBN flake cross sections taken with screw dislocation imaging conditions (top) and edge imaging conditions (bottom). Defects only observed in the top image are screw dislocations, while those only observed in the bottom are edge type. Dislocations present in both are mixed-type, as they have components of both screw and edge dislocations. 